Article

\title{
In Vitro Selective Growth-Inhibitory Activities of Phytochemicals, Synthetic Phytochemical Analogs, and Antibiotics against Diarrheagenic/Probiotic Bacteria and Cancer/Normal Intestinal Cells
}

\author{
Tomas Kudera $^{1}$, Ivo Doskocil ${ }^{2}\left(\mathbb{D}\right.$, Hana Salmonova $^{2}\left(\mathbb{D}\right.$, Miloslav Petrtyl $^{3}$, Eva Skrivanova ${ }^{2}$ \\ and Ladislav Kokoska ${ }^{1, *}$ \\ 1 Department of Crop Sciences and Agroforestry, Faculty of Tropical AgriSciences, \\ Czech University of Life Sciences Prague, Kamycka 129, 16500 Praha-Suchdol, Czech Republic; \\ kuderat@ftz.czu.cz \\ 2 Department of Microbiology, Nutrition and Dietetics, Faculty of Agrobiology, Food and Natural Resources, \\ Czech University of Life Sciences Prague, Kamycka 129, 16500 Praha-Suchdol, Czech Republic; \\ doskocil@af.czu.cz (I.D.); salmonova@af.czu.cz (H.S.); skrivanovae@af.czu.cz (E.S.) \\ 3 Department of Zoology and Fisheries, Faculty of Agrobiology, Food and Natural Resources, \\ Czech University of Life Sciences Prague, Kamycka 129, 16500 Praha-Suchdol, Czech Republic; \\ petrtyl@af.czu.cz \\ * Correspondence: kokoska@ftz.czu.cz; Tel.: +420-224-382-180
}

Received: 21 July 2020; Accepted: 31 August 2020; Published: 3 September 2020

\begin{abstract}
A desirable attribute of novel antimicrobial agents for bacterial diarrhea is decreased toxicity toward host intestinal microbiota. In addition, gut dysbiosis is associated with an increased risk of developing intestinal cancer. In this study, the selective growth-inhibitory activities of ten phytochemicals and their synthetic analogs (berberine, bismuth subsalicylate, ferron, 8-hydroxyquinoline, chloroxine, nitroxoline, salicylic acid, sanguinarine, tannic acid, and zinc pyrithione), as well as those of six commercial antibiotics (ceftriaxone, ciprofloxacin, chloramphenicol, metronidazole, tetracycline, and vancomycin) against 21 intestinal pathogenic/probiotic (e.g., Salmonella spp. and bifidobacteria) bacterial strains and three intestinal cancer/normal (Caco-2, HT29, and FHs 74 Int) cell lines were examined in vitro using the broth microdilution method and thiazolyl blue tetrazolium bromide assay. Chloroxine, ciprofloxacin, nitroxoline, tetracycline, and zinc pyrithione exhibited the most potent selective growth-inhibitory activity against pathogens, whereas 8-hydroxyquinoline, chloroxine, nitroxoline, sanguinarine, and zinc pyrithione exhibited the highest cytotoxic activity against cancer cells. None of the tested antibiotics were cytotoxic to normal cells, whereas 8-hydroxyquinoline and sanguinarine exhibited selective antiproliferative activity against cancer cells. These findings indicate that 8-hydroxyquinoline alkaloids and metal-pyridine derivative complexes are chemical structures derived from plants with potential bioactive properties in terms of selective antibacterial and anticancer activities against diarrheagenic bacteria and intestinal cancer cells.
\end{abstract}

Keywords: plant compounds; diarrhea; antibacterial; anticancer; selectivity

\section{Introduction}

The lack of an effective and safe antimicrobial therapy for diarrheagenic bacterial infections is a global health concern, especially in developing countries for children under the age of five years [1]. Although mortality associated with bacterial diarrhea is low in developed countries, the increased incidence rates of inflammatory bowel disease and colorectal cancer have also been attributed to 
gut dysbiosis that can result from chronic intestinal infections [2]. Currently, infectious diarrhea is treated using conventional drugs belonging to various classes of antibiotics, such as ceftriaxone, chloramphenicol, ciprofloxacin, tetracycline, metronidazole, and vancomycin. However, the irrational use of antibiotics, including incorrect dose prescription, has led to the development of drug resistance in several pathogens. Additionally, the applications of conventional antibiotics are limited, especially among children in developing countries, owing to high cost and increased risk of side effects including gut dysbiosis. Therefore, there is a need to identify novel antimicrobial agents for infectious bacterial diarrhea to overcome the limitations of conventional antimicrobial drugs [3].

Over the last decades, plant-derived products have become a mainstay in providing novel chemical scaffolds for the development of anti-infective drugs, and therefore antidiarrheal medicinal plants and their bioactive components can be examined first [4]. However, the therapeutic effect of products derived from antidiarrheal medicinal plants is not necessarily based on their antimicrobial activity against the causative agents as other mechanisms can be considered important, such as antimotility and antisecretory effects, for their useful applications [5]. The chance of finding a new plant-derived compound with promising antibacterial activity could therefore be enhanced by the chemotaxonomic approach by examining such novel medicinal plants that are taxonomically related to the species known to bear specific types of phytochemicals with growth-inhibitory effect on diarrheagenic bacteria [6]. In a recent review paper, Kokoska et al. [7] described the in vitro antimicrobial properties and clinical efficacy of plant-derived compounds with their synthetic analogs that are present in products available in the international market as over-the-counter pharmaceuticals, dietary supplements, and herbal medicines for intestinal infections. Benzylisoquinoline alkaloid berberine (e.g., Hydrastis canadensis), simple phenol bismuth subsalicylate, the analog of salicylic acid derived from salicin (Salix alba), and polyphenol tannic acid (e.g., Quercus spp.) were mentioned as examples of the efficient agents with potent activity against some of the gut bacterial pathogens.

As it has recently been proposed, investigations of new antimicrobial agents should be focused on the identification of structures with lowered toxicity to indigenous intestinal microbiota including probiotic bacterial strains. Since there is a common association between dysbiosis and the use of antibiotics, the agents selectively acting against pathogenic microorganisms can prevent the risk of developing diseases, such as chronic bowel inflammation and intestinal carcinoma [2]. Although the composition of the whole gut microbiota comprises a large number of microorganisms forming a complex ecosystem, a preliminary screening of selective antibacterial activity of newly tested agents could be performed by testing the in vitro susceptibilities of particular representatives of each of the three dominant bacterial phyla that can be found in human intestines, and which probiotic function have been described. Among them, we can recognize the strains such as Bifidobacterium spp. (Actinobacteria), Lactobacillus spp. (Firmicutes) and Bacteroides fragilis (Bacteroidetes) [8,9]. Selective in vitro growth inhibitory effect of a plant-derived compound was for example described in the study of Novakova et al. [10], where anticlostridial effect of 8-hydroxyquinoline (Microstachys corniculata) was comparably higher than the activities revealed against different strains of bifidobacteria. Chloroxine, the synthetic 8-hydroxyquinoline derivative, is the antimicrobial agent that has also been used as an oral formulation for infectious diarrhea, and disorders of the intestinal microbiota [11]. In dysbiosis, the increased abundance of Fusobacterium nucleatum and Faecalibacterium prausnitzii is positively and negatively correlated with the risk of intestinal carcinogenesis, respectively [12]. The repression of gut microbiota enhances the susceptibility of host intestinal cells to diarrheagenic bacterial infection and chemical-induced cytotoxicity. Thus, novel antimicrobial agents should not exhibit cytotoxic activity against normal intestinal cells [13]. For example, quinolone antibiotics were reported to inhibit the growth of both bacterial and eukaryotic cells through the same mechanism and consequently enhance the risk of eliciting cytotoxic response [14,15]. On the other hand, the antiproliferative activity of these agents with selective cytotoxic effects against intestinal cancer cells would be a suitable feature in cases of dysbiosis associated with carcinogenesis. There are limited studies on the anticancer effects of conventional antibiotics. For example, Bourikas et al. [16] reported that ciprofloxacin is potent 
to inhibit the proliferation of intestinal cancer cell line HT29 in vitro. On the other hand, a strong in vitro antiproliferative activity of plant-derived compounds against various cancer cell lines has extensively been reported, and some are used as a scaffold for anticancer drugs. For example, the alkaloid camptothecin, extracted from the bark of Camptotheca acuminata, is currently used as a cytostatic agent for the treatment of colon cancer [17]. We, therefore, suggest that the phytochemicals with known in vitro growth-inhibitory activity against some of the diarrheagenic bacteria could potentially exhibit selective cytotoxic effects on cancer cells. Amongst them, the anticancer effect has generally been reported for quinoline alkaloids [18]. In addition to those earlier mentioned, benzylisoquinoline alkaloid sanguinarine (Sanguinaria canadensis) [7,19], and 8-hydroxyquinoline derivatives ferron [20] and nitroxoline [21] are examples of antimicrobial drugs with potent anticancer activity. Another type of synthetic phytochemical analog with reported antimicrobial and anticancer properties is metal-pyridine derivative complex zinc pyrithione (pyrithione found in Polyalthia nemoralis) [7,22].

In this study, we compared the selective antibacterial (diarrheagenic/probiotic strains) and cytotoxic (cancer/normal intestinal cells) activities of phytochemicals (alkaloids and phenolics) and their synthetic analogs with those of antibiotics in vitro. For each test compound, values of minimum inhibitory concentrations (MICs), half-maximal inhibitory concentration ( $\mathrm{IC}_{50}$ ), and $80 \%$ inhibitory concentration of proliferation $\left(\mathrm{IC}_{80}\right)$ were assessed. The means of these values $\left(\bar{x}\right.$-MIC, $\bar{x}-\mathrm{IC}_{50}$, and $\left.\bar{x}-\mathrm{IC}_{80}\right)$, each defined for a particular type of strain/cell line, were used for calculation of selectivity index (SI) between activities against normal intestinal cells and diarrheagenic strains (SIa), probiotic and diarrheagenic strains (SIb), and normal and cancer intestinal cells (SIc). The aim was to obtain some of the missing data on particular in vitro activities of the test compound, to assist in identifying phytochemicals with scaffolds that possess a potent combination of bioactivities. They could subsequently be utilized in future chemotaxonomic investigation of antidiarrheal medicinal plants with their bioactive components considered for new chemotherapeutic agents against diarrheal infections and associated intestinal cancer diseases. Our results show that 8-hydroxyquinoline alkaloids and zinc pyrithione possess in vitro selective antibacterial properties against diarrheagenic bacteria comparable to ciprofloxacin and tetracycline, with additional in vitro antiproliferative activity against cancer intestinal cell lines. However, in contrary to antibiotics, these compounds generally possess increased cytotoxicity to normal intestinal cells.

\section{Results}

\subsection{Antibacterial Activity}

As far as the antibacterial activity of antibiotics against diarrheagenic strains is considered, ciprofloxacin and tetracycline exhibited strong growth-inhibitory effect ( $\bar{x}$-MICs $=2 \pm 4$ and $4.8 \pm$ $8 \mu \mathrm{g} / \mathrm{mL}$, respectively), while chloramphenicol and ceftriaxone exhibited moderate growth-inhibitory activities ( $\bar{x}$-MIC $=16.5 \pm 34$ and $61.8 \pm 141 \mu \mathrm{g} / \mathrm{mL}$, respectively). Regarding the significant degree of variation between MICs of these compounds, particular types of pathogenic strains were highly susceptible while some other species were distinctly more resistant. For example, all gram-negative diarrheagenic bacteria were highly susceptible to ciprofloxacin (MICs $=0.016-0.125 \mu \mathrm{g} / \mathrm{mL}$ ) and ceftriaxone (MICs $=0.062-0.5 \mu \mathrm{g} / \mathrm{mL}$ ). In contrast, their MICs produced against gram-positive pathogens were comparably higher; for the former ranging from 1 to $16 \mu \mathrm{g} / \mathrm{mL}$ and for the latter in the range of $4-512 \mu \mathrm{g} / \mathrm{mL}$. At least half of the diarrheagenic bacteria were inhibited at the low MICs $(1-4 \mu \mathrm{g} / \mathrm{mL})$ by chloramphenicol and tetracycline, whereas the variations were particularly caused by the weak activities revealed against Enterococcus faecalis (MIC $=128 \mu \mathrm{g} / \mathrm{mL}$ ) and Clostridium perfringens ( $32 \mu \mathrm{g} / \mathrm{mL}$ ), respectively. At the low concentration (MICs $=0.5 \mu \mathrm{g} / \mathrm{mL}$ ), tetracycline also inhibited Clostridium difficile and Bacillus cereus. Although metronidazole and vancomycin were generally inactive against diarrheagenic bacteria $(\bar{x}$-MIC $=651.4 \pm 459$ and $512.9 \pm 404$, respectively), they produced a strong inhibitory effect against both clostridial species tested (MICs $=0.5-8 \mu \mathrm{g} / \mathrm{mL}$ ), whereas the former also exhibited strong activity against Escherichia coli $(0.062 \mu \mathrm{g} / \mathrm{mL})$. The synthetic 
analogs of phytochemicals, namely, zinc pyrithione, nitroxoline, and chloroxine, exhibited strong to moderate growth-inhibitory activity against all diarrheagenic bacteria $(\bar{x}$-MICs $=7.1 \pm 4,12 \pm 10$, and $24 \pm 19 \mu \mathrm{g} / \mathrm{mL}$, respectively). The activities of these compounds against particular pathogenic bacteria did not have significant differences, however, some of the strains were comparably more susceptible. Regarding that, B. cereus, E. coli, Shigella flexneri, and Vibrio parahaemolyticus were highly susceptible to zinc pyrithione (MICs $=1-4 \mu \mathrm{g} / \mathrm{mL}$ ). The MIC of chloroxine against both B. cereus and C. difficile was $8 \mu \mathrm{g} / \mathrm{mL}$. Nitroxoline exhibited strong growth-inhibitory activities (MICs $=2-4 \mu \mathrm{g} / \mathrm{mL}$ ) against B. cereus, clostridial species, E. coli, and S. flexneri. Although 8-hydroxyquinoline did not produce significant antibacterial activity against the pathogens $(\bar{x}$-MIC $=224.4 \pm 181)$, its growth-inhibitory activities were strong against $E$. faecalis (MIC $=4 \mu \mathrm{g} / \mathrm{mL}$ ) and Listeria monocytogenes (MIC $=1 \mu \mathrm{g} / \mathrm{mL}$ ).

Subsequently, the growth-inhibitory activities of different test compounds against probiotic strains were evaluated. Probiotic bacteria exhibited high susceptibility to chloramphenicol $(\bar{x}$-MIC $=6.2$ $\pm 4 \mu \mathrm{g} / \mathrm{mL}$ ) and medium susceptibility to tetracycline, nitroxoline, zinc pyrithione, ciprofloxacin, ceftriaxone, sanguinarine, and vancomycin $(\bar{x}$-MICs $=19.8 \pm 19-47.8 \pm 78 \mu \mathrm{g} / \mathrm{mL})$. The MICs $(2-4 \mu \mathrm{g} / \mathrm{mL})$ of both chloramphenicol and vancomycin against bifidobacteria were similarly low, but the latter produced significantly lower activities against lactobacilli (MICs $=64-256 \mu \mathrm{g} / \mathrm{mL}$ ) and B. fragilis $(\mathrm{MIC}=32 \mu \mathrm{g} / \mathrm{mL}$ ). With the exception of Bifidobacterium breve and Bifidobacterium longum ssp. longum $(\mathrm{MICs}=8-32 \mu \mathrm{g} / \mathrm{mL})$, the remaining bifidobacteria were also susceptible to ceftriaxone (MICs = $1-4 \mu \mathrm{g} / \mathrm{mL}$ ). However, considering the MIC range (MICs $=0.5-32 \mu \mathrm{g} / \mathrm{mL}$ ) shown against lactobacilli and the low activity against $B$. fragilis (MIC $=128 \mu \mathrm{g} / \mathrm{mL}$ ), the variation of activities of this drug against probiotic bacteria is quite high. The exceptionally strong activities against $B$. fragilis were revealed by metronidazole and tetracycline (MICs $=0.5 \mu \mathrm{g} / \mathrm{mL}$ ), whereas the MIC $(4 \mu \mathrm{g} / \mathrm{mL}$ ) of nitroxoline against this bacterium was the same as in the case of chloramphenicol. In general, berberine (MICs $\geq$ $32 \mu \mathrm{g} / \mathrm{mL}$ ), ferron (MICs $\geq 64 \mu \mathrm{g} / \mathrm{mL}$ ), and phenolic compounds (MICs $\geq 64 \mu \mathrm{g} / \mathrm{mL}$ ) did not exhibit significant antibacterial activities against any of the 21 strains. The complete data on growth-inhibitory activities of test compounds against diarrheagenic and probiotic strains, including calculated mean values $(\bar{x}$-MIC) are presented in Table 1 . 
Table 1. In vitro selective inhibitory activities of phytochemicals, their synthetic analogs, and antibiotics against intestinal bacteria and cells.

\begin{tabular}{|c|c|c|c|c|c|c|c|c|c|c|c|c|c|c|c|c|c|}
\hline \multirow{2}{*}{\multicolumn{2}{|c|}{ Cultures Tested }} & \multicolumn{7}{|c|}{ Alkaloids } & \multicolumn{3}{|c|}{ Phenolic Compounds } & \multicolumn{6}{|c|}{ Antibiotics } \\
\hline & & BR & SG & $8 \mathrm{HQ}$ & CLX & NXL & FRN & $\mathbf{Z P}$ & SA & BS & TA & CF & $\mathrm{CP}$ & MA & VM & CA & TC \\
\hline \multirow{23}{*}{ 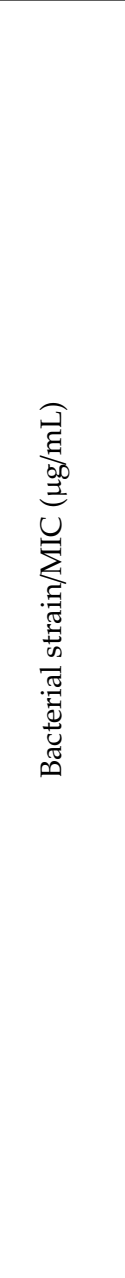 } & $\mathrm{BC}$ & $-a^{a}$ & 128 & 512 & 8 & 4 & 512 & 4 & - & 512 & 512 & 128 & 1 & 128 & 256 & 8 & 0.5 \\
\hline & $\mathrm{CD}$ & - & 64 & 128 & 8 & 2 & 64 & 8 & 256 & 128 & - & 64 & 16 & 0.5 & 2 & 4 & 0.5 \\
\hline & $\mathrm{CP}$ & 256 & 128 & 128 & 16 & 4 & 64 & 8 & - & 512 & 512 & 4 & 1 & 8 & 1 & 4 & 32 \\
\hline & $\mathrm{EF}$ & - & 32 & 4 & 16 & 32 & - & 8 & - & - & - & 512 & 2 & - & - & 128 & 4 \\
\hline & $\mathrm{EC}$ & - & 256 & 128 & 16 & 4 & - & 4 & - & - & - & 0.062 & 0.062 & 0.062 & - & 4 & 1 \\
\hline & ECS & - & 128 & 256 & 64 & 32 & - & 8 & - & - & 512 & 0.5 & 0.016 & - & 512 & 8 & 4 \\
\hline & $\mathrm{LM}$ & - & 16 & 1 & 32 & 16 & 512 & 8 & - & 256 & - & 32 & 4 & 512 & 8 & 8 & 2 \\
\hline & SF & - & 64 & 128 & 16 & 2 & - & 1 & - & - & - & 0.5 & 0.016 & - & - & 4 & 2 \\
\hline & $\mathrm{SE}$ & - & 256 & 256 & 64 & 16 & - & 8 & - & - & - & 0.25 & 0.031 & - & 512 & 4 & 4 \\
\hline & ST & 512 & 512 & 512 & 16 & 16 & - & 8 & - & 512 & - & 0.25 & 0.031 & - & 512 & 8 & 4 \\
\hline & VP & 512 & 32 & 128 & 16 & 8 & 256 & 4 & - & - & 128 & 0.125 & 0.062 & - & 256 & 2 & 1 \\
\hline & YE & - & 256 & 512 & 16 & 8 & - & 16 & - & - & 512 & 0.25 & 0.125 & - & - & 16 & 2 \\
\hline & $\begin{array}{l}\bar{x} \text {-DB } \\
\pm \mathrm{SD}\end{array}$ & $\begin{array}{l}874.7 \\
\pm 256 \\
\end{array}$ & $\begin{array}{c}156 \pm \\
137\end{array}$ & $\begin{array}{l}224.4 \\
\pm 181 \\
\end{array}$ & $\begin{array}{c}24 \pm \\
19\end{array}$ & $\begin{array}{c}12 \pm \\
10\end{array}$ & $\begin{array}{l}714.7 \\
\pm 388 \\
\end{array}$ & $\begin{array}{c}7.1 \pm \\
4\end{array}$ & $\begin{array}{c}960 \pm \\
212\end{array}$ & $\begin{array}{l}757.3 \\
\pm 332 \\
\end{array}$ & $\begin{array}{l}778.7 \\
\pm 307 \\
\end{array}$ & $\begin{array}{c}61.8 \pm \\
141\end{array}$ & $2 \pm 4$ & $\begin{array}{r}651.4 \\
\pm 459 \\
\end{array}$ & $\begin{array}{l}512.9 \\
\pm 404 \\
\end{array}$ & $\begin{array}{c}16.5 \pm \\
34\end{array}$ & $\begin{array}{c}4.8 \pm \\
8\end{array}$ \\
\hline & $\mathrm{BF}$ & - & 32 & 32 & 32 & 4 & 128 & 8 & 128 & 32 & - & 128 & 8 & 0.5 & 32 & 4 & 0.5 \\
\hline & $\mathrm{BA}$ & 128 & 16 & 512 & 128 & 16 & 256 & 16 & - & 32 & - & 1 & 8 & 64 & 2 & 4 & 64 \\
\hline & BLC & 32 & 32 & - & 512 & 32 & 512 & 16 & 128 & 64 & - & 2 & 32 & 32 & 2 & 4 & 32 \\
\hline & $\mathrm{BBF}$ & 64 & 32 & 512 & - & 16 & 512 & 8 & - & 64 & - & 4 & 16 & - & 4 & 4 & 16 \\
\hline & $\mathrm{BB}$ & 64 & 32 & - & 64 & 32 & 512 & 16 & - & 64 & 256 & 32 & 64 & 32 & 4 & 4 & 16 \\
\hline & $\mathrm{BL}$ & 32 & 64 & 512 & 64 & 16 & 256 & 16 & 512 & 64 & 128 & 8 & 16 & 8 & 2 & 4 & 2 \\
\hline & LC & 64 & 32 & - & 128 & 32 & 256 & 16 & 512 & 128 & 512 & 32 & 32 & - & 256 & 16 & 8 \\
\hline & LR & - & 32 & - & 128 & 16 & 512 & 64 & 512 & 64 & 512 & 0.5 & 32 & 256 & 64 & 8 & 32 \\
\hline & LRM & 64 & 64 & - & 128 & 16 & 512 & 32 & 512 & 128 & - & 32 & 4 & - & 64 & 8 & 8 \\
\hline & $\begin{array}{l}\bar{x}-\mathrm{PB} \\
\pm \mathrm{SD}\end{array}$ & $\begin{array}{l}277.3 \\
\pm 400\end{array}$ & $\begin{array}{c}37.3 \pm \\
15\end{array}$ & $\begin{array}{l}743.1 \\
\pm 343\end{array}$ & $\begin{array}{l}245.3 \\
\pm 306\end{array}$ & $20 \pm 9$ & $\begin{array}{c}384 \pm \\
148\end{array}$ & $\begin{array}{c}21.3 \pm \\
16\end{array}$ & $\begin{array}{l}597.3 \\
\pm 336\end{array}$ & $\begin{array}{c}71.1 \pm \\
33\end{array}$ & $\begin{array}{l}725.3 \\
\pm 352\end{array}$ & $\begin{array}{c}26.6 \pm \\
38\end{array}$ & $\begin{array}{c}23.6 \pm \\
18\end{array}$ & $\begin{array}{l}384.9 \\
\pm 458\end{array}$ & $\begin{array}{c}47.8 \pm \\
78\end{array}$ & $\begin{array}{c}6.2 \pm \\
4\end{array}$ & $\begin{array}{c}19.8 \pm \\
19\end{array}$ \\
\hline
\end{tabular}


Table 1. Cont.

\begin{tabular}{|c|c|c|c|c|c|c|c|c|c|c|c|c|c|c|c|c|c|c|}
\hline \multirow{2}{*}{\multicolumn{3}{|c|}{ Cultures Tested }} & \multicolumn{7}{|c|}{ Alkaloids } & \multicolumn{3}{|c|}{ Phenolic Compounds } & \multicolumn{6}{|c|}{ Antibiotics } \\
\hline & & & BR & SG & $8 \mathrm{HQ}$ & CLX & NXL & FRN & $\mathbf{Z P}$ & SA & BS & TA & $\mathrm{CF}$ & $\mathrm{CP}$ & MA & VM & CA & TC \\
\hline \multirow{8}{*}{ 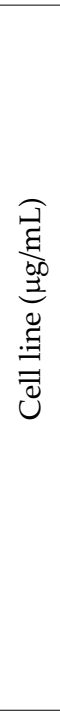 } & \multirow{8}{*}{$\begin{array}{l}0 \\
\text { क } \\
+1 \\
0 \\
0 \\
0 \\
0 \\
0 \\
+1 \\
0\end{array}$} & HT29 & $5 \pm 1$ & $\begin{array}{c}0.9 \pm \\
0.2\end{array}$ & $\begin{array}{c}1.3 \pm \\
0.3\end{array}$ & $\begin{array}{c}3.7 \pm \\
0.3\end{array}$ & $\begin{array}{c}2.6 \pm \\
0.3\end{array}$ & $\begin{array}{c}86.3 \pm \\
12\end{array}$ & $\begin{array}{c}0.6 \pm \\
0.01\end{array}$ & - & $\begin{array}{l}461.9 \\
\pm 17\end{array}$ & $\begin{array}{c}35.9 \pm \\
4.9\end{array}$ & - & $\begin{array}{l}130.3 \\
\pm 13\end{array}$ & - & - & $\begin{array}{c}271.1 \\
\pm 1\end{array}$ & $\begin{array}{c}392.9 \pm \\
20\end{array}$ \\
\hline & & Caco-2 & $\begin{array}{c}19.4 \pm \\
2.9\end{array}$ & $\begin{array}{c}0.8 \pm \\
0.1\end{array}$ & $\begin{array}{c}0.3 \pm \\
0.1\end{array}$ & $\begin{array}{c}1.3 \pm \\
0.04\end{array}$ & $\begin{array}{c}1.1 \pm \\
0.03\end{array}$ & $\begin{array}{c}55.2 \pm \\
4.8\end{array}$ & $\begin{array}{c}0.7 \pm \\
0.2\end{array}$ & - & $\begin{array}{c}45.3 \pm \\
5\end{array}$ & $\begin{array}{c}27.6 \pm \\
1.5\end{array}$ & - & $\begin{array}{c}69.9 \pm \\
4.9\end{array}$ & - & - & $\begin{array}{c}439.3 \\
\pm 4\end{array}$ & $\begin{array}{c}70.4 \pm \\
15\end{array}$ \\
\hline & & $\begin{array}{l}\bar{x}-\mathrm{CC} \\
\pm \mathrm{SD}\end{array}$ & $\begin{array}{c}12.2 \pm \\
7\end{array}$ & $\begin{array}{c}0.8 \pm \\
0.05\end{array}$ & $\begin{array}{c}0.8 \pm \\
0.5\end{array}$ & $\begin{array}{c}2.5 \pm \\
1.2\end{array}$ & $\begin{array}{c}1.8 \pm \\
0.8\end{array}$ & $\begin{array}{c}70.8 \pm \\
16\end{array}$ & $\begin{array}{c}0.6 \pm \\
0.05\end{array}$ & - & $\begin{array}{l}253.6 \\
\pm 208\end{array}$ & $\begin{array}{c}31.7 \pm \\
4\end{array}$ & - & $\begin{array}{c}100.1 \\
\pm 30\end{array}$ & - & - & $\begin{array}{c}355.2 \\
\pm 84\end{array}$ & $\begin{array}{c}231.6 \pm \\
161\end{array}$ \\
\hline & & $\begin{array}{c}\text { FHs } \\
74 \text { Int }\end{array}$ & $\begin{array}{l}1 \pm \\
0.1\end{array}$ & $\begin{array}{l}1 \pm \\
0.1\end{array}$ & $\begin{array}{c}10.7 \pm \\
0.2\end{array}$ & $\begin{array}{c}0.5 \pm \\
0.02\end{array}$ & $\begin{array}{c}0.4 \pm \\
0.05\end{array}$ & $\begin{array}{c}22.6 \pm \\
3.3\end{array}$ & $\begin{array}{c}0.3 \pm \\
0.1\end{array}$ & $\begin{array}{c}73.2 \pm \\
4.6\end{array}$ & $\begin{array}{c}8.7 \pm \\
1.3\end{array}$ & $\begin{array}{c}5.9 \pm \\
1.2\end{array}$ & - & $\begin{array}{c}51.8 \pm \\
27\end{array}$ & - & - & $\begin{array}{c}30.7 \pm \\
5.6\end{array}$ & $\begin{array}{c}14.7 \pm \\
2.3\end{array}$ \\
\hline & & HT29 & $\begin{array}{c}42.1 \pm \\
7.3\end{array}$ & $\begin{array}{c}1.8 \pm \\
0.4\end{array}$ & $\begin{array}{c}4.8 \pm \\
2.8\end{array}$ & $\begin{array}{l}5 \pm \\
0.7\end{array}$ & $\begin{array}{c}3.7 \pm \\
0.4\end{array}$ & $\begin{array}{c}149.4 \\
\pm 6\end{array}$ & $\begin{array}{c}0.7 \pm \\
0.01\end{array}$ & - & - & $\begin{array}{c}43 \pm \\
2.2\end{array}$ & - & - & - & - & - & - \\
\hline & & Caco-2 & $\begin{array}{c}78.9 \pm \\
0.3\end{array}$ & $\begin{array}{c}1.5 \pm \\
0.1\end{array}$ & $\begin{array}{c}0.9 \pm \\
0.1\end{array}$ & $\begin{array}{c}5.9 \pm \\
0.7\end{array}$ & $\begin{array}{l}5 \pm \\
1.5\end{array}$ & $\begin{array}{l}139.9 \\
\pm 26\end{array}$ & $\begin{array}{c}0.7 \pm \\
0.01\end{array}$ & - & $\begin{array}{c}454.9 \\
\pm 43\end{array}$ & - & - & - & - & - & - & - \\
\hline & & $\begin{array}{l}\bar{x}-\mathrm{CC} \\
\pm \mathrm{SD}\end{array}$ & $\begin{array}{c}60.5 \pm \\
18.4\end{array}$ & $\begin{array}{c}1.6 \pm \\
0.2\end{array}$ & $\begin{array}{c}2.9 \pm \\
2\end{array}$ & $\begin{array}{c}5.4 \pm \\
0.5\end{array}$ & $\begin{array}{c}4.4 \pm \\
0.7\end{array}$ & $\begin{array}{l}144.7 \\
\pm 4.8\end{array}$ & $\begin{array}{c}0.7 \pm \\
0\end{array}$ & - & $\begin{array}{c}740 \pm \\
285\end{array}$ & $\begin{array}{c}534 \pm \\
491\end{array}$ & - & - & - & - & - & - \\
\hline & & $\begin{array}{l}\text { FHs } \\
74 \mathrm{Int}\end{array}$ & $\begin{array}{c}26.4 \pm \\
0.8\end{array}$ & $\begin{array}{c}1.9 \pm \\
0.2\end{array}$ & $\begin{array}{c}20.3 \pm \\
2.4\end{array}$ & $\begin{array}{l}2 \pm \\
0.1\end{array}$ & $\begin{array}{c}0.8 \pm \\
0.05\end{array}$ & $\begin{array}{c}46 \pm \\
2.2\end{array}$ & $\begin{array}{c}0.5 \pm \\
0.03\end{array}$ & $\begin{array}{c}206.9 \\
\pm 69\end{array}$ & $\begin{array}{c}31.4 \pm \\
6.6\end{array}$ & $\begin{array}{c}10.2 \pm \\
2.4\end{array}$ & - & $\begin{array}{c}129.5 \\
\pm 24\end{array}$ & - & - & - & $\begin{array}{c}108.2 \\
\pm 5\end{array}$ \\
\hline \multirow{3}{*}{\multicolumn{2}{|c|}{$\bar{s}$}} & (a) & -1.5 & -1.9 & -1 & -1.1 & -1.2 & -1.2 & -1.1 & -0.7 & -1.4 & -1.9 & 1.2 & 1.8 & 0.2 & 0.3 & 1.8 & 1.4 \\
\hline & & (b) & -0.5 & -0.6 & 0.5 & 1 & 0.2 & -0.3 & 0.5 & -0.2 & -1 & -0.03 & -0.4 & 1.1 & -0.2 & -1 & -0.4 & 0.6 \\
\hline & & (c) & -1.1 & 0.1 & 1.1 & -0.7 & -0.6 & -0.5 & -0.4 & -1.1 & -1.5 & -0.7 & 0 & -0.3 & 0 & 0 & -1.1 & -1.2 \\
\hline
\end{tabular}

MIC: minimum inhibitory concentration; $\mathrm{IC}_{50}$ : half maximal inhibitory concentration; $\mathrm{IC}_{80}: 80 \%$ inhibitory concentration of proliferation; $\mathrm{SD}$. standard deviation. ${ }^{\text {a }}$ Not active (MIC/IC ${ }_{50 / 80}$ $>512 \mu \mathrm{g} / \mathrm{mL}$, the value $1024 \mu \mathrm{g} / \mathrm{mL}$ was used for average calculation). BR: berberine, SG: sanguinarine, 8HQ: 8-hydroxyquinoline, CLX: chloroxine, NXL: nitroxoline, FRN: ferron, ZP: zinc pyrithione, SA: salicylic acid, BS: bismuth subsalicylate, TA: tannic acid, CF: ceftriaxone, CP: ciprofloxacin, MA: metronidazole, VM: vancomycin, CA: chloramphenicol, TC: tetracycline. BC: Bacillus cereus, CD: Clostridium difficile, CP: Clostridium perfringens, EF: Enterococcus faecalis, EC: Escherichia coli, ECS: E. coli 0175:H7, LM: Listeria monocytogenes, SF: Shigella flexneri, SE: Salmonella Enteritidis, ST: Salmonella Typhimurium, VP: Vibrio parahaemolyticus, YE: Yersinia enterocolitica, BF: Bacteroides fragilis, BA: Bifidobacterium adolescentis, BLC: Bifidobacterium animalis spp. lactis, BBF: Bifidobacterium bifidum, BB: Bifidobacterium breve, BL: Bifidobacterium longum ssp. longum, LC: Lactobacillus casei, LR: Lactobacillus reuteri, LRM: Lactobacillus rhamnosus.

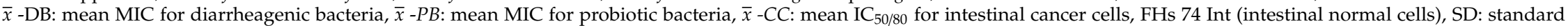
deviation. SI (Selective Index): (a) normal cells/diarrheagenic bacteria, (b) probiotic bacteria/diarrheagenic bacteria, (c) normal cells/cancer cells 


\subsection{Cytotoxic Effect}

Amongst the test compounds, only alkaloids and related structures exhibited strong cytotoxic activity, while other agents, especially antibiotics, exhibited moderate or no cytotoxic activity. Considering the antiproliferative effect of antibiotics on normal intestinal cells (FHs 74 Int), ceftriaxone, metronidazole, and vancomycin were not cytotoxic at all tested concentrations $\left(\mathrm{IC}_{50}\right.$ and $\mathrm{IC}_{80}>$ $512 \mu \mathrm{g} / \mathrm{mL}$ ), whereas tetracycline ( $\mathrm{IC}_{50}=14.7 \pm 2.3 \mu \mathrm{g} / \mathrm{mL} ; \mathrm{IC}_{80}=108.2 \pm 5 \mu \mathrm{g} / \mathrm{mL}$ ), chloramphenicol $\left(\mathrm{IC}_{50}=30.7 \pm 5.6 \mu \mathrm{g} / \mathrm{mL} ; \mathrm{IC}_{80}>512 \mu \mathrm{g} / \mathrm{mL}\right)$, and ciprofloxacin $\left(\mathrm{IC}_{50}=51.8 \pm 27 \mu \mathrm{g} / \mathrm{mL} ; \mathrm{IC}_{80}=\right.$ $129.5 \pm 24 \mu \mathrm{g} / \mathrm{mL}$ ) were moderately cytotoxic. In case of phytochemicals and their synthetic analogs, salicylic acid $\left(\mathrm{IC}_{50}=73.2 \pm 4.6 \mu \mathrm{g} / \mathrm{mL} ; \mathrm{IC}_{80}=206.9 \pm 69 \mu \mathrm{g} / \mathrm{mL}\right)$, ferron $\left(\mathrm{IC}_{50}=22.6 \pm 3.3 \mu \mathrm{g} / \mathrm{mL}\right.$; $\mathrm{IC}_{80}=46 \pm 2.2 \mu \mathrm{g} / \mathrm{mL}$ ), and 8-hydroxyquinoline $\left(\mathrm{IC}_{50}=10.7 \pm 0.2 \mu \mathrm{g} / \mathrm{mL} ; \mathrm{IC}_{80}=20.3 \pm 2.4 \mu \mathrm{g} / \mathrm{mL}\right)$, revealed moderately cytotoxic effects against FHs $74 \mathrm{Int}$, whereas the other compounds were cytotoxic $\left(\mathrm{IC}_{50}\right.$ values $=0.3 \pm 0.1-1 \pm 0.1 \mu \mathrm{g} / \mathrm{mL} ; \mathrm{IC}_{80}$ values $\left.=0.5 \pm 0.03-26.4 \pm 0.8 \mu \mathrm{g} / \mathrm{mL}\right)$. Considering the antiproliferative effect on cancer intestinal cells, zinc pyrithione, 8-hydroxyquinoline, and sanguinarine were cytotoxic to HT29 ( $\mathrm{IC}_{50}$ values $=0.6,1.3$, and $0.9 \mu \mathrm{g} / \mathrm{mL}$, respectively) and Caco-2 ( $\mathrm{IC}_{50}$ values $=0.7,0.3$ and $0.8 \mu \mathrm{g} / \mathrm{mL}$, respectively) cells. Nitroxoline $\left(\mathrm{IC}_{50}=1.1 \mu \mathrm{g} / \mathrm{mL}\right)$ and chloroxine $\left(\mathrm{IC}_{50}=\right.$ $1.3 \mu \mathrm{g} / \mathrm{mL}$ ) exhibited comparable cytotoxic activity against Caco-2 cells. Zinc pyrithione had the lowest $\bar{x}$-IC 50 value $(0.6 \pm 0.05 \mu \mathrm{g} / \mathrm{mL})$ against cancer cells, followed by 8 -hydroxyquinoline $(0.8 \pm 0.5 \mu \mathrm{g} / \mathrm{mL})$, sanguinarine $(0.8 \pm 0.05 \mu \mathrm{g} / \mathrm{mL})$, nitroxoline $(1.8 \pm 0.8 \mu \mathrm{g} / \mathrm{mL})$, and chloroxine $(2.5 \pm 1.2 \mu \mathrm{g} / \mathrm{mL})$. Berberine $\left(\bar{x}-\mathrm{IC}_{50}=12.2 \pm 7 \mu \mathrm{g} / \mathrm{mL}\right)$, tannic acid $\left(\bar{x}-\mathrm{IC}_{50}=31.7 \pm 4 \mu \mathrm{g} / \mathrm{mL}\right)$, and ferron $\left(\bar{x}-\mathrm{IC}_{50}=70.8 \pm\right.$ $16 \mu \mathrm{g} / \mathrm{mL})$, produced moderate cytotoxic activity, while salicylic acid and bismuth subsalicylate $\left(\bar{x}\right.$-IC $\mathrm{I}_{50}$ $\geq 253.6 \pm 208 \mu \mathrm{g} / \mathrm{mL}$ ) did not exhibit significant cytotoxic activity against cancer cells. At relatively high concentrations, some antibiotics exhibited antiproliferative activity against cancer cells, namely: ciprofloxacin, tetracycline, and chloramphenicol $\left(\bar{x}-\mathrm{IC}_{50}=100.1 \pm 30-355.2 \pm 84 \mu \mathrm{g} / \mathrm{mL}\right)$. The complete data on the antiproliferative activities of test compounds against normal and cancer intestinal cells, including calculated mean values for the latter $\left(\bar{x}-\mathrm{IC}_{50}\right.$ and $\left.\bar{x}-\mathrm{IC}_{80}\right)$, are presented in Table 1.

\subsection{Selective Toxicity}

The selective antibacterial activities against the pathogens with relatively lower activity against probiotic strains (SIb values range from 0.2-1.1) was revealed by most of the agents exhibiting strong to moderate inhibitory effects on diarrheagenic bacteria, namely: nitroxoline, zinc pyrithione, tetracycline, chloroxine, and ciprofloxacin. In contrast, chloramphenicol and ceftriaxone were more toxic to probiotic strains (SIbs $=-0.4$ for both). Although the antibacterial activity of berberine, ferron, phenolic compounds, and sanguinarine was in cases of both diarrheagenic and probiotic strains generally insignificant, the results show that these agents were rather toxic to the latter (SIb values range from -1 to -0.03). Due to the minor cytotoxicity revealed against FHs 74 Int, none of the antibiotics exhibited an increased toxicity to normal intestinal cells at the inhibitory concentrations active against diarrheagenic bacteria (SIa values $=0.2-1.8$ ), especially ciprofloxacin and chloramphenicol. In contrast, all of the phytochemicals and their synthetic analogs revealed cytotoxicity to normal intestinal cells at the concentrations they were generally inactive against diarrheagenic bacteria (SIa values range from -1.9 to -0.7$)$. Only 8-hydroxyquinoline (SIc $=1.1$ ) and sanguinarine (SIc $=0.1$ ) exhibited selective antiproliferative activity against cancer cells with the decreased cytotoxic effect on normal intestinal cells. Except these two, other tested compounds were more toxic to normal than to cancer intestinal cells (SIcs $=$ from -1.5 to -0.3 ), or in the case of ceftriaxone, metronidazole, and vancomycin, they did not show any selectivity (SIcs $=0$ ), as they did not inhibit cell lines at any concentration tested. The data on selective toxicities, including all calculated SI values are presented in Table 1. The curves of in vitro selective concentration-dependent effect of ciprofloxacin, chloroxine, nitroxoline, tetracycline, and zinc pyrithione on the growth of diarrheagenic and probiotic bacteria and of 8-hydroxyquinoline on intestinal normal and cancer cells proliferation are shown in Figure 1. 

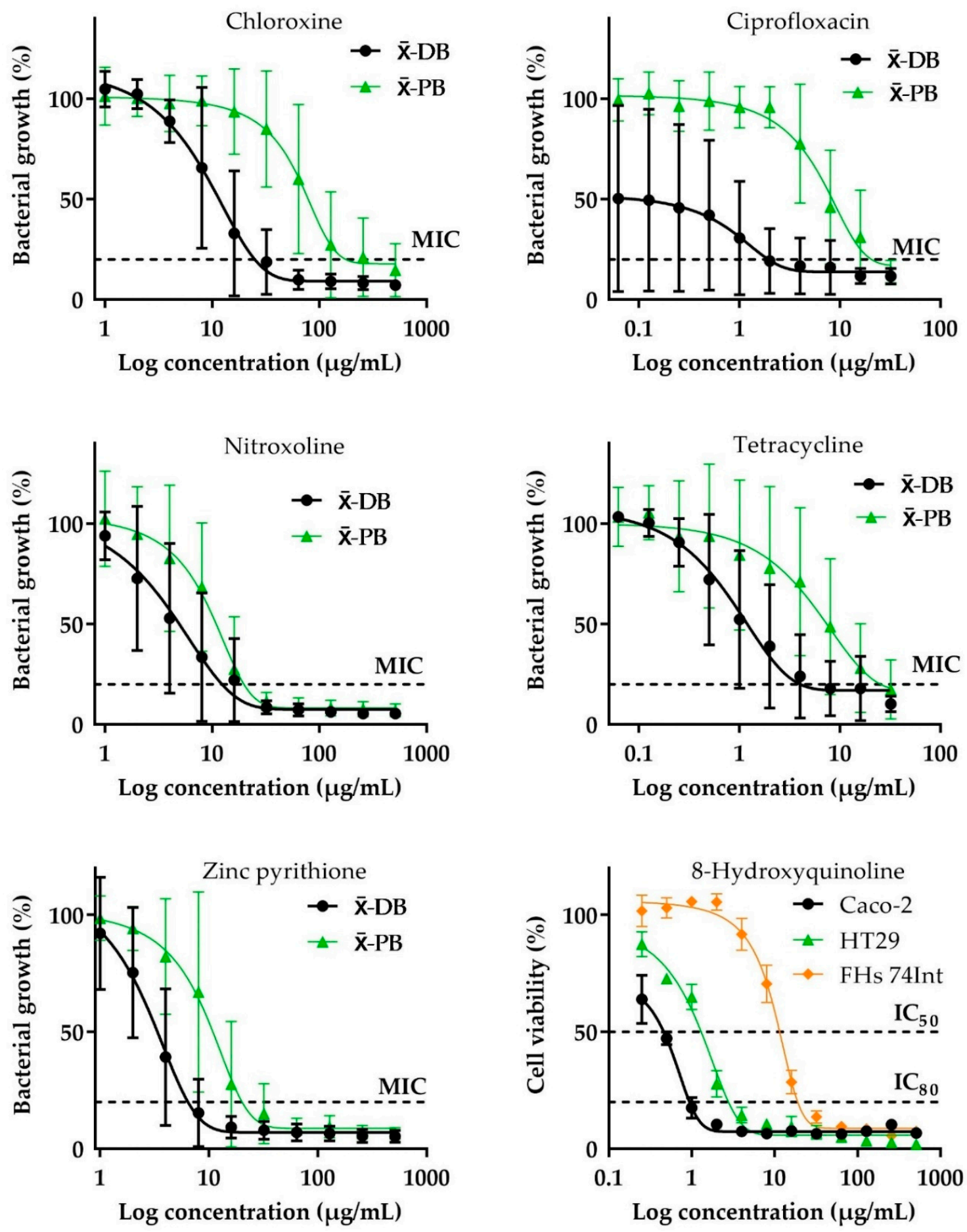

Figure 1. Selective concentration-dependent effect of chloroxine, ciprofloxacin, nitroxoline, tetracycline, and zinc pyrithione on the growth of diarrheagenic and probiotic bacteria and of 8-hydroxyquinoline on intestinal normal and cancer cells proliferation in vitro. MIC: minimum inhibitory concentration; $\mathrm{IC}_{50}$ : half maximal inhibitory concentration; $\mathrm{IC}_{80}: 80 \%$ inhibitory concentration of proliferation. $\bar{x}$-DB: mean MIC for 12 diarrheagenic bacteria, $\bar{x}$-PB: mean MIC for 9 probiotic bacteria; Caco-2 and HT29: intestinal cancer cells; Fhs74 Int: intestinal normal cells. 


\subsection{Principal Component Analysis (PCA)}

The correlation between biological activities and chemical structures of the tested compounds and their groups (antibiotics, phenolic compounds, alkaloids, and related structures) was analyzed using PCA (Figure 2). Although the compounds were distributed equally in all quadrants, detailed analysis revealed specific patterns. The closest correlation was observed in the lower-left quadrant between four antibiotics (ceftriaxone, ciprofloxacin, chloramphenicol, and tetracycline), which indicated that these antibiotics exhibited strong growth-inhibitory activity against diarrheagenic and probiotic strains but did not exhibit distinct cytotoxic activity against normal and cancer intestinal cells. The second highest correlation observed in the lower right quadrant indicated that zinc pyrithione and 8-hydroxyquinolines (excluding ferron) exhibited selective antibacterial activity against diarrheagenic strains along with strong to moderate cytotoxic activity against both types of tested cell lines. The correlation observed in the upper right quadrant indicated that tannic acid, benzylisoquinoline alkaloids, and ferron exhibited moderate to no antipathogenic effect (with negative SIbs) and overall moderate to strong cytotoxic activity. The upper left quadrant contains the remaining antibiotics (metronidazole and vancomycin) and both simple phenols with minimal correlation. These agents exhibited moderate to no growth-inhibiting activity against diarrheagenic strains and practically no cytotoxic activity. Whereas all alkaloids are distributed in lower and upper right quadrants indicating their capability to reveal any type of the tested bioactivities, phenols are spread in the right and left upper quadrants which shows their lack of significant antibacterial activity but a certain degree of cytotoxicity. In contrast, antibiotics are concentrated in the lower-left quadrant slightly overlapping the upper left one, therefore they usually display a significant antibacterial effect which is rarely accompanied by cytotoxicity.

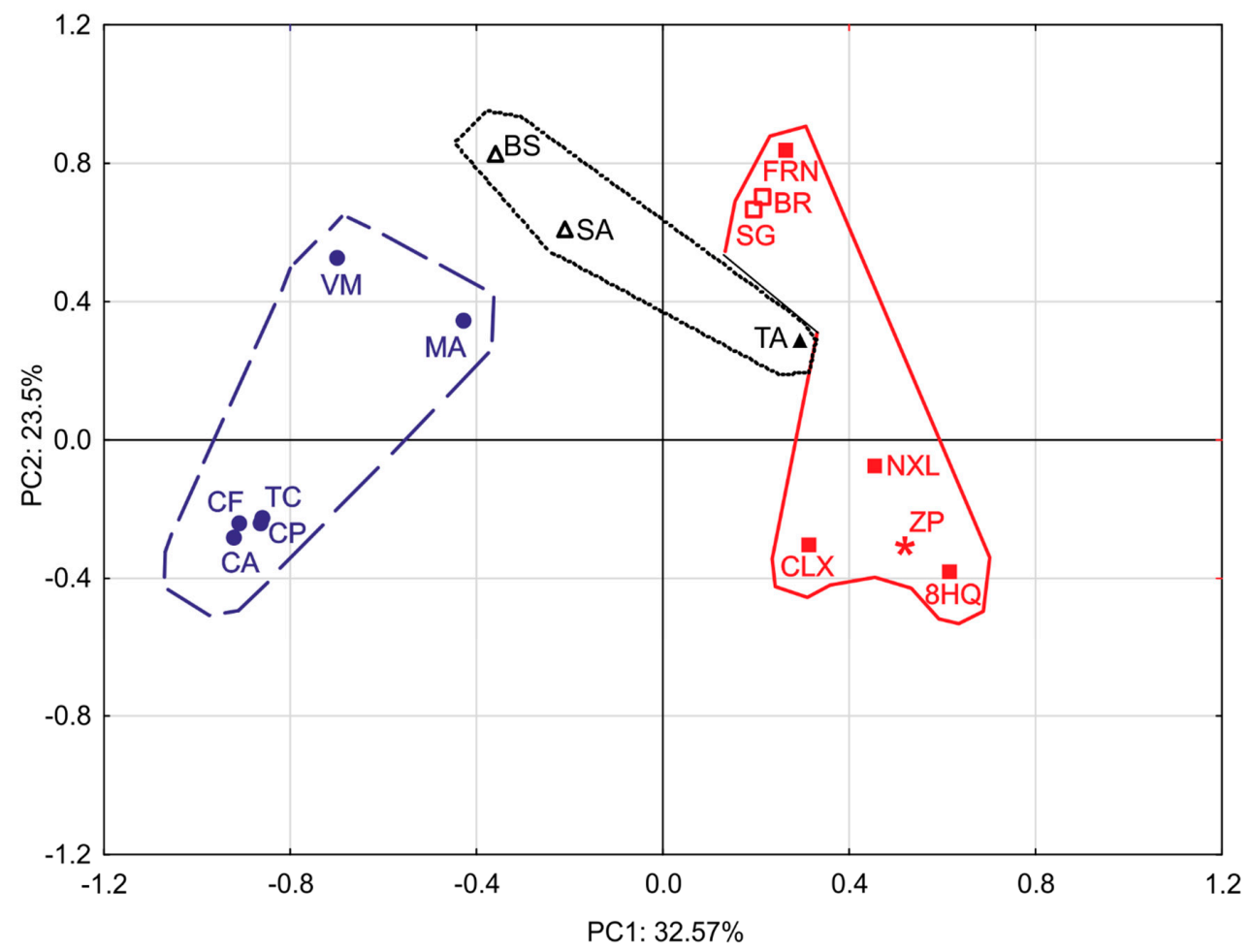

Figure 2. Principal component analysis of antibacterial and cytotoxic activities of phytochemicals, their synthetic analogues, and antibiotics against intestinal bacteria and cells in vitro. BR: berberine, SG: sanguinarine, 8HQ: 8-hydroxyquinoline, CLX: chloroxine, NXL: nitroxoline, FRN: ferron, ZP: zinc pyrithione, SA: salicylic acid, BS: bismuth subsalicylate, TA: tannic acid, CF: ceftriaxone, CP: ciprofloxacin, MA: metronidazole, VM: vancomycin, CA: chloramphenicol, TC: tetracycline. Antibiotics $[-, \bullet]$; Phenolic compounds [ $\cdots \cdots$, poly- $(\mathbf{\Lambda})$, simple $(\boldsymbol{\Delta})]$; Alkaloids and related structures [benzylisoquinolines $(\square)$, 8-hydroxyquinolines $(\square)$, metal-pyridine derivative complex $\left(^{*}\right)$. 


\section{Discussion}

The in vitro growth-inhibitory properties of the tested antibiotics have previously been reported for a number of diarrheagenic and probiotic bacteria. However, the bacterial strains tested as well as the methods with criteria used for antimicrobial activity assessment vary frequently among the previous studies. Moreover, the studies reporting the in vitro susceptibilities of probiotic bacteria deal more with clinical isolates [23], and less with standard strains [24]. The present study, therefore, provides the data on in vitro selective antibacterial activities of these antibiotics that can be fairly compared with the same data obtained for phytochemicals and their synthetic analogs. We suggest that the reason behind the increased resistance of probiotic strains differ for particular compounds that showed a selective antipathogenic effect. The growth-inhibitory activities of fluoroquinolones against Gram-positive bacteria are reported to be lower than those against Gram-negative bacteria [15]. Consistent with this finding, bifidobacteria and lactobacilli (Gram-positive) were generally less susceptible to ciprofloxacin than Gram-negative diarrheagenic bacteria that predominate over Gram-positive pathogens in this study. The decreased susceptibility of bifidobacteria to tetracycline might be caused by the presence of specific antibiotic resistance genes [25]. Similar to other third-generation cephalosporins [26], the growth-inhibitory activity of ceftriaxone against Gram-negative bacteria was higher than that against Gram-positive bacteria. However, as a result of significant resistance of the tested Gram-positive pathogens and susceptibility of bifidobacteria, ceftriaxone showed increased toxicity to probiotic strains. The growth-inhibitory activities of some alkaloids and related structures were comparable with those of antibiotics. The antibacterial activity of 8-hydroxyquinoline alkaloids is mediated through the chelation of metals that function as co-factors in various enzymes, which results in the inhibition of RNA synthesis. We suggest that probiotic strains (mainly bifidobacteria) are more resistant to 8-hydroxyquinolines as they are able to sequester iron from the environment [10]. The selective antibacterial activity of 8-hydroxyquinoline against diarrheagenic pathogens seems to be enhanced with chlorine halogenation or by the presence of a nitro group and decreased with iodine halogenation and the presence of a sulfo group, as respectively observed for chloroxine, nitroxoline, and ferron in our study. The in vitro selective anticlostridial effect of 8-hydroxyquinoline with increased resistance of bifidobacteria was previously described in studies of Novakova et al. [10,27,28], Skrivanova et al. [29], and Kim et al. [30]. However, data on its in vitro growth-inhibitory effects against a broader selection of diarrheagenic bacteria are limited. The present study also provides new data on in vitro antibacterial activities of chloroxine against diarrheagenic bacteria in addition to those previously published [31,32]. It has been reported that Endiaron, a chloroxine-containing antimicrobial product used for infectious diarrhea, exhibits antimicrobial activity against the pathogens and does not affect the host indigenous microbiota [11], which is in agreement with the increased resistance of probiotic bacteria described in the present study. Interestingly, the antipathogenic activity of nitroxoline, used to treat urinary tract infections, was higher than that of chloroxine. However, the antibacterial selectivity of nitroxoline against diarrheagenic strains was lower. Out of the intestinal bacteria tested herein, there are only data on in vitro inhibitory effects of nitroxoline against $E$. coli and E. faecalis that have been reported before [33]. In spite of zinc pyrithione being only used topically for dermatological infections [7], in relation to the plant compounds and their synthetic analogs in this study, it exhibited the highest growth-inhibitory activity against diarrheagenic bacteria with lowered toxicity to probiotic bacteria. According to our best knowledge, this is the first report on in vitro selective antibacterial activities of zinc pyrithione on intestinal diarrheagenic and probiotic bacteria. Although there is limited knowledge on the mechanism underlying the antibacterial activity of zinc pyrithione, the mechanism may be similar to that of 8-hydroxyquinolines [34]. The weak antimicrobial activities of phenols against diarrheagenic strains are consistent with those reported in previous studies. The effectiveness of phenols in infectious diarrhea may be based on other mechanisms, such as astringent, mucosa-protective, and anti-inflammatory properties, or inhibition of pathogenic enterotoxins [7]. Although clinical studies on extensively used phytochemical berberine have reported comparably higher efficiency than certain antibiotics (e.g., chloramphenicol) [35], our results did not show its significant in vitro 
antibacterial activity. A possible reason for this discordance is that berberine rather neutralizes diarrheagenic action of bacteria by inhibiting their enterotoxins, as described by Sack and Froelich [36]. The MICs of both benzylisoquinoline alkaloids against some diarrheagenic bacteria reported in this study were higher than those reported in previous studies. This may be because the inoculum density used in this study was higher than that used in previous studies [7,37].

The mechanism underlying the antiproliferative activity of some antibiotics, such as ciprofloxacin, tetracycline, and chloramphenicol, may be similar to that underlying antimicrobial activity $[14,38,39]$. Previous studies have evaluated the antiproliferative activity of chemicals derived from ciprofloxacin and tetracycline against cancer cells and suggested their applications in cancer therapy $[14,40]$. Consistent with the results of this study, previous studies have revealed that other antibiotics are not cytotoxic to eukaryotic cells [41-43]. The antitumor activities of some plant compounds and their synthetic analogs have been investigated previously. In the case of 8-hydroxyquinoline and its derivatives, the interaction with metal ions, namely copper and iron, and their transportation into cells has been reported as crucial for its antiproliferative activity [44]. Freitas et al. [45] reported that 8-hydroxyquinoline derivatives with potent anticancer potential often contain halogen substituents. However, in the present study, nitroxoline exhibited stronger antiproliferative activity against cancer cells than chloroxine and ferron. Previous studies have reported that the underlying mechanism of antitumor activity of zinc pyrithione and sanguinarine involves the inhibition of proteasomal deubiquitinases and microtubule depolymerization, respectively [19,22]. All of the above-mentioned alkaloids and related structures revealed increased toxicity to normal intestinal cells in comparison with their antipathogenic effect, which limits their applications in treating bacterial diarrhea. Although there are no studies reporting oral toxicity or toxicity to the digestive system from berberine, chloroxine, and nitroxoline, we suggest that their safety profile should be further examined and the potential protective role of indigenous gut microbiota against these cytotoxic chemicals should be more deeply studied. Zinc pyrithione, 8-hydroxyquinoline, and sanguinarine are not part of any product intended for internal use. Hence, their dose-dependent toxicological profile and oral safety must be carefully elucidated before any consideration for their application for treating infectious diarrhea associated with intestinal cancer [46].

\section{Materials and Methods}

\subsection{Chemicals}

Phytochemicals (berberine chloride, 8-hydroxyquinoline, salicylic acid, tannic acid, and sanguinarine chloride) and their synthetic analogs [chloroxine (5,7-dichloroquinolin-8-ol), nitroxoline (5-nitroquinolin-8-ol), ferron (7-iodo-8-hydroxyquinoline-5-sulfonic acid), bismuth subsalicylate, and zinc pyrithione], as well as antibiotics (ceftriaxone sodium, ciprofloxacin, chloramphenicol, metronidazole, tetracycline, and vancomycin hydrochloride), used in this study were purchased from Sigma-Aldrich (Prague, Czech Republic). Dimethyl sulfoxide (DMSO) (Sigma-Aldrich, Prague, Czech Republic) was used to prepare the stock solutions of all test compounds, except those of metronidazole, salicylic acid, vancomycin, and zinc pyrithione, which were prepared using distilled water. Stock solutions of chloramphenicol, tannic acid, and tetracycline were prepared using $96 \%$ ethanol (Sigma-Aldrich, Prague, Czech Republic). The chemical structures of individual compounds tested are shown in Figure 3. 
<smiles></smiles>

1<smiles>O=C(O)c1ccccc1O</smiles>

8<smiles></smiles>

2<smiles>O=C(O[B]O)c1ccccc1O</smiles>

9<smiles>[R]c1cc([R])c2cccnc2c1O</smiles>

$3 \mathrm{R}_{1}=\mathrm{R}_{2}=\mathrm{H}$

$4 \mathrm{R}_{1}=\mathrm{R}_{2}=\mathrm{Cl}$

$5 \mathrm{R}_{1}=\mathrm{NO}_{2}, \mathrm{R}_{2}=\mathrm{H}$

$6 \mathrm{R}_{1}=\mathrm{SO}_{3} \mathrm{H}, \mathrm{R}_{2}=\mathrm{I}$<smiles>[O-][Z16](Sc1cccc[n+]1[O-])Sc1cccc[n+]1[O-]</smiles>

7<smiles>COCC(=O)c1cc(O)c(O)c(O)c1</smiles><smiles>O=C([O-])c1cc(O)c(O)c(O)c1</smiles><smiles>CONC(C(=O)NC1C(=O)N2C(CSc3nc(=O)c(=O)[nH]n3C)=C(C(=O)O)CSC12)c1csc(N)n1</smiles>

11<smiles>COc1cc(C(=O)Oc2cc(C(=O)O)cc(OC(=O)c3cc(O)c(O)c(OC(=O)c4cc(O)c(O)c(OC(=O)c5cc(O)c(O)c(O)c5)c4)c3)c2OC(=O)c2cc(O)c(O)c(OC(=O)c3cc(O)c(O)c(CCc4cc(O)c(O)c(O)c4)c3)c2OC(=O)c2cc(O)c(O)c(O)c2)cc(O)c1O</smiles>

10<smiles>O=C(O)c1cn(C2CC2)c2cc(N3CCNCC3)c(F)cc2c1=O</smiles>

12<smiles>Cc1ncc([N+](=O)[O-])n1CCO</smiles><smiles>O=C(N[C@@H](CO)C(O)c1ccc([N+](=O)[O-])cc1)C(Cl)Cl</smiles>

15

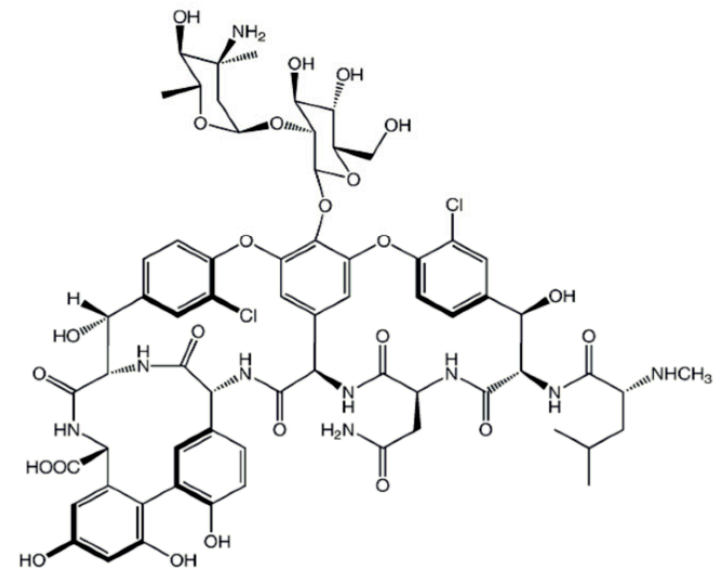

14<smiles>CN(C)C1C(O)=C(C(N)=O)C(=O)C2(O)C(O)=C3C(=O)c4c(O)cccc4[C@@](C)(O)C3CC12</smiles>

16

Figure 3. The chemical structures of the tested phytochemicals, their synthetic analogs, and antidiarrheal antibiotics. 1. berberine chloride, 2. sanguinarine chloride, 3. 8-hydroxyquinoline, 4. chloroxine, 5. nitroxoline, 6. ferron, 7. zinc pyrithione, 8. salicylic acid, 9. bismuth subsalicylate, 10. tannic acid, 11. ceftriaxone sodium, 12. ciprofloxacin, 13. metronidazole, 14. vancomycin hydrochloride, 15. chloramphenicol, 16 . tetracycline.

\subsection{Bacterial Strains and Growth Media}

The intestinal bacterial type strains were obtained from the American Type Culture Collection (ATCC, Rockville, MD, USA), Czech Collection of Microorganisms (CCM, Brno, Czech Republic), German Collection of Microorganisms and Cell Cultures (DSMZ, Braunschweig, Germany), and National Collection of Type Cultures (NCTC, London, UK). In accordance with the diversity of diarrheagenic Gram-positive and Gram-negative bacteria responsible for globally distributed 
foodborne, waterborne, and nosocomial infections [3,47], the following 12 strains were used in this study: B. cereus (ATCC 14579), C. difficile (DSMZ 12056), C. perfringens (DSMZ 11778), E. faecalis (ATCC 29212), E. coli (ATCC 25922), E. coli 0175:H7 (NCTC 12900), L. monocytogenes (ATCC 7644), S. flexneri (ATCC 12022), Salmonella enterica ssp. enterica serovar Enteritidis (ATCC 13076), S. enterica ssp. enterica serovar Typhimurium (ATCC 14028), V. parahaemolyticus (ATCC 17802), and Yersinia enterocolitica (ATCC 9610). The following nine bacterial strains, which belong to three predominant bacterial phyla in the human gut and exhibit probiotic functions [8,9], were used in this study: Bacteroides fragilis (ATCC 25285), Bifidobacterium adolescentis (DSMZ 20087), Bifidobacterium animalis spp. lactis (DSMZ 10140), Bifidobacterium bifidum (ATCC 29521), B. breve (ATCC 15700), B. longum ssp. longum (DSMZ 20219), Lactobacillus casei (DSMZ 20011), Lactobacillus reuteri (CCM 3625), and Lactobacillus rhamnosus (CCM 7091). As the maintenance and growth medium, Mueller-Hinton broth (Oxoid, Basingstoke, UK) was used for bacteria that grow aerobically (E. faecalis supp. 1\% glucose, V. parahaemolyticus supp. 3\% $\mathrm{NaCl}$ ). The anaerobic bacteria (clostridia and bifidobacteria), including facultative species (lactobacilli), were cultured in Wilkins-Chalgren broth (Oxoid, Basingstoke, UK) supplemented with $5 \mathrm{~g} / \mathrm{L}$ soya peptone and $0.5 \mathrm{~g} / \mathrm{L}$ cysteine.

\subsection{Cell Cultures}

One representative normal intestinal cell line (FHs 74 Int (ATCC CCL 241)) and two cancer intestinal cell lines (Caco-2 (ATCC HTB 37) and HT29 (ATCC HTB 38)) were purchased from ATCC (Rockville, MD, USA). Normal cells were cultured in Hybri-Care medium supplemented with 10\% fetal bovine serum, $1 \%$ sodium bicarbonate, $1 \%$ non-essential amino acids, $30 \mathrm{ng} / \mathrm{mL}$ of epidermal growth factor, and $1 \%$ penicillin-streptomycin solution $(10,000$ units $/ \mathrm{mL}$ and $100 \mathrm{mg} / \mathrm{mL}$, respectively). The cancer cells were cultured in Dulbecco's modified Eagle's medium (DMEM) supplemented with $1 \%$ sodium pyruvate, $10 \%$ fetal bovine serum, $1 \%$ sodium bicarbonate, $1 \%$ non-essential amino acids, and $1 \%$ penicillin-streptomycin solution $(10,000$ units $/ \mathrm{mL}$ and $100 \mathrm{mg} / \mathrm{mL}$, respectively) (all purchased from Sigma-Aldrich, Prague, Czech Republic). The cultures were incubated at $37^{\circ} \mathrm{C}$ and $5 \% \mathrm{CO}_{2}$. The culture medium was replaced every $2-3$ days and cells were passaged every 7 days.

\subsection{Antibacterial Assay}

The growth-inhibitory activities of the test compounds against aerobic and anaerobic bacterial strains were evaluated by the broth microdilution method using 96-well microtiter plates, following the protocols of CLSI guidelines [48] and Hecht et al. [49], respectively. Prior to testing, the strains were sub-cultured in the appropriate media at $37^{\circ} \mathrm{C}$ for $24 \mathrm{~h}$. Obligate anaerobes and lactobacilli were cultured for $48 \mathrm{~h}$ using Whitley A35 Anaerobic Workstation (Don Whitley Scientific, Bingley, UK). The anaerobic conditions were created by the supply of a standard anaerobic gas mixture of $10 \% \mathrm{H}_{2}, 10 \% \mathrm{CO}_{2}$, and $80 \% \mathrm{~N}_{2}$ (Linde Gas, Prague, Czech Republic). Test agents were diluted 2-fold in appropriate growth media using the Freedom EVO 100 automated pipetting platform (Tecan, Männedorf, Switzerland) or multichannel pipette (Eppendorf, Hamburg, Germany) (initial concentration of $512 \mu \mathrm{g} / \mathrm{mL}$ ). After the bacterial cultures reached an inoculum density of $1.5 \times 10^{8} \mathrm{CFU} / \mathrm{mL}$ by $0.5 \mathrm{McF}$ arland standard using Densi-La-Meter II (Lachema, Brno, Czech Republic), the 96-well plates were inoculated ( $5 \mu \mathrm{L} /$ well). The plates containing the volatile compound, 8-hydroxyquinoline, were covered using EVA capmats (Micronic, Lelystad, Netherlands) after inoculation to prevent evaporation [50]. Bacterial cultures in microplates were incubated by employing the same protocols as used for their cultivation prior to the test. The optical density of the cultures was measured at $405 \mathrm{~nm}\left(\mathrm{OD}_{450 \mathrm{~nm}}\right)$ using a Cytation 3 Imaging Reader (BioTek, Winooski, VT, USA) before and after the growth. The lowest concentration $(\mu \mathrm{g} / \mathrm{mL})$ of test compounds at which the bacterial growth was inhibited by $\geq 80 \%$ was defined as MIC. All tests were performed as three independent experiments each carried out in triplicate. The data are presented as median/mode. As a result of experiments performed without dissolved test compounds, DMSO and 96\% ethanol (both from Sigma-Aldrich, Prague, Czech Republic) did not inhibit bacterial growth of any strain at the tested concentrations $(\leq 1 \%)$. 


\subsection{Cytotoxicity Assay}

The antiproliferative activities of test compounds against normal and cancer intestinal lines were evaluated using the modified thiazolyl blue tetrazolium bromide (MTT) cytotoxicity assay developed by Mosmann et al. [51]. The cancer $\left(2.5 \times 10^{3}\right)$ and normal intestinal $\left(2.5 \times 10^{5}\right)$ cells were seeded in a 96-well microtiter plate for $24 \mathrm{~h}$. Cells were incubated with two-fold serially diluted test compounds $(0.25-512 \mu \mathrm{g} / \mathrm{mL})$ for $72 \mathrm{~h}$. Plates containing 8-hydroxyquinoline were covered using EVA capmats. Next, the cells were incubated with MTT reagent $(1 \mathrm{mg} / \mathrm{mL}$ ) (Sigma-Aldrich, Prague, Czech Republic) in DMEM or Hybri-Care medium for an additional $2 \mathrm{~h}$ at $37^{\circ} \mathrm{C}$ and $5 \% \mathrm{CO}_{2}$. The medium with MTT was removed and the intracellular formazan product was dissolved in $100 \mu \mathrm{L}$ of DMSO. The absorbance was measured at $555 \mathrm{~nm}$ using a Tecan Infinite M200 spectrometer (Tecan, Männedorf, Switzerland), and the percentage of viability was calculated when compared to an untreated control. Antiproliferative activity of the test compounds was represented as $\mathrm{IC}_{50}(\mu \mathrm{g} / \mathrm{mL})$. Three independent experiments (two replicates each) were performed for every test. Data are presented as mean \pm standard deviation. DMSO was used as a positive control at the highest concentration with and without EVA capmats. The solvents did not affect the viability of normal and cancer intestinal cell lines at the tested concentration $(\leq 1 \%)$.

\subsection{Calculations and Statistics}

For comparison of microbiological and toxicological data, $\mathrm{IC}_{80}$ was calculated as equivalent to the MIC endpoint, defined as $80 \%$ bacterial growth inhibition [52]. Subsequently, $\bar{x}$-MIC, $\bar{x}$-IC $\mathrm{IC}_{50}$, and $\bar{x}$-IC 80 values ( \pm standard deviations) were calculated to quantify the inhibitory activity of test compounds against diarrheagenic/probiotic bacteria and cancer cells, respectively. After that, SIa (normal intestinal cells/ diarrheagenic strains), SIb (probiotic/diarrheagenic strains), and SIc (normal/cancer intestinal cells) was calculated using the formulas below.

$$
\begin{aligned}
& \text { SIa }=\log \left(X_{1} / Y_{1}\right), \\
& \text { SIb }=\log \left(X_{2} / Y_{1}\right) \\
& \text { SIc }=\log \left(X_{3} / Y_{2}\right)
\end{aligned}
$$

where: $\mathrm{X}_{1}=\mathrm{IC}_{80}$ against FHs $74 \mathrm{Int} ; \mathrm{X}_{2}=\bar{x}$-MIC against probiotic strains; $\mathrm{X}_{3}=\mathrm{IC}_{50}$ against FHs $74 \mathrm{Int} ; \mathrm{Y}_{1}=\bar{x}$-MIC against diarrheagenic strains; $\mathrm{Y}_{2}=\bar{x}$-IC $\mathrm{IC}_{50}$ against cancer intestinal cells. The SI values $>0$ and $<0$ indicate selective toxicity against diarrheagenic strains/cancer cell lines and probiotic strains/normal cell lines, respectively.

The correlation between the combination of activities revealed by test compounds and their chemical classes was analyzed using PCA with Statistica 13 software [53]. All data for particular activities were grouped into four types of targets (cancer cells, diarrheagenic strains, normal cells, and probiotic strains) using MIC and $\mathrm{IC}_{80}$ values. There was no adjustment of PCA parameters for this analysis. For the calculation of each $\bar{x}$-MIC, $\bar{x}$ - $\mathrm{IC}_{50}, \bar{x}$ - $\mathrm{IC}_{80}$ and for PCA, values greater than the maximum tested concentration $(512 \mu \mathrm{g} / \mathrm{mL})$ were replaced with $1024 \mu \mathrm{g} / \mathrm{mL}$.

\section{Conclusions}

In summary, ciprofloxacin, 8-hydroxyquinoline alkaloids (chloroxine and nitroxoline), tetracycline, and zinc pyrithione exhibited a significant selective growth-inhibitory activity against diarrheagenic bacteria with lowered toxicity to probiotic bacteria in vitro. 8-Hydroxyquinoline, chloroxine, nitroxoline, sanguinarine, and zinc pyrithione also exhibited a strong cytotoxic effect, whereas the antiproliferative action of 8-hydroxyquinoline and sanguinarine were selective to cancer intestinal cells. These findings indicate that 8-hydroxyquinoline alkaloids and metal-pyridine derivative complexes are chemical structures with promising bioactive properties in terms of in vitro selective antibacterial and anticancer activities which could be utilized in future chemotaxonomic investigation of antidiarrheal medicinal 
plants and their bioactive components. These could be further investigated as possible new chemotherapeutic agents against diarrheal infections and associated intestinal cancer diseases. However, in vivo studies on the toxicity of these compounds with more complex animal models will be needed before their consideration to be used for this purpose.

Author Contributions: Conceptualization, T.K. and L.K.; methodology, T.K., L.K., and I.D.; software, M.P.; validation, L.K., I.D., H.S., M.P., and E.S.; formal analysis, T.K., L.K., I.D., H.S., M.P., and E.S.; investigation, T.K., L.K., I.D., H.S., and E.S.; resources, L.K., H.S., and E.S.; data curation, T.K., L.K., I.D., and M.P.; writing-original draft preparation, T.K. and L.K.; writing-review and editing, I.D., H.S., M.P., and E.S.; visualization, T.K. and M.P.; supervision, L.K.; project administration, L.K.; funding acquisition, L.K. and E.S. All authors have read and agreed to the published version of the manuscript.

Funding: This research was funded by the Czech University of Life Sciences Prague, project IGA 20205001, and European Regional Development Fund, project CZ.02.1.01/0.0/0.0/16_019/0000845.

Conflicts of Interest: The authors declare no conflict of interest.

\section{References}

1. World Health Organization. Diarrhoeal Disease. Available online: https://www.who.int/en/news-room/factsheets/detail/diarrhoeal-disease (accessed on 19 March 2020).

2. Garrett, W.S. The gut microbiota and colon cancer. Science 2019, 364, 1133-1135. [CrossRef] [PubMed]

3. Diniz-Santos, D.R.; Silva, L.R.; Silva, N. Antibiotics for the empirical treatment of acute infectious diarrhea in children. Braz. J. Infect. Dis. 2006, 10, 217-227. [CrossRef] [PubMed]

4. White, N.J. Qinghaosu (Artemisinin): The Price of Success. Science 2008, 320, 330-334. [CrossRef] [PubMed]

5. Formiga, R.D.O.; Quirino, Z.G.M.; Diniz, M.D.F.F.M.; Marinho, A.F.; Tavares, J.F.; Batista, L. Maytenus Erythroxylon Reissek (Celastraceae) ethanol extract presents antidiarrheal activity via antimotility and antisecretory mechanisms. World J. Gastroenterol. 2017, 23, 4381-4389. [CrossRef] [PubMed]

6. Hao, D.C.; Xiao, P.G. Pharmaceutical resource discovery from traditional medicinal plants: Pharmacophylogeny and pharmacophylogenomics. Chin. Herb. Med. 2020, 12, 104-117. [CrossRef]

7. Kokoska, L.; Kloucek, P.; Leuner, O.; Novy, P. Plant-Derived Products as Antibacterial and Antifungal Agents in Human Health Care. Curr. Med. Chem. 2019, 26, 5501-5541. [CrossRef]

8. Sun, F.; Zhang, Q.; Zhao, J.; Zhang, H.; Zhai, Q.; Chen, W. A potential species of next-generation probiotics? The dark and light sides of Bacteroides fragilis in health. Food Res. Int. 2019, 126, 108590. [CrossRef]

9. Behnsen, J.; Deriu, E.; Sassone-Corsi, M.; Raffatellu, M. Probiotics: Properties, Examples, and Specific Applications. Cold Spring Harb. Perspect. Med. 2013, 3, a010074. [CrossRef]

10. Novakova, J.; Vlkova, E.; Bonusova, B.; Rada, V.; Kokoska, L. In vitro selective inhibitory effect of 8-hydroxyquinoline against bifidoba cteria and clostridia. Anaerobe 2013, 22, 134-136. [CrossRef]

11. WikiZero: Chloroxine. Available online: https://wikizero.com/en/Chloroxine (accessed on 10 August 2020).

12. Bruneau, A.; Baylatry, M.T.; Joly, A.C.; Sokol, H. Gut microbiota: What impact on colorectal carcinogenesis and treatment? Bull. Cancer 2018, 105, 70-80. [CrossRef]

13. Sambruy, Y.; Ferruzza, S.; Ranaldi, G.; De Angelis, I. Intestinal cell culture models: Applications in toxicology and pharmacology. Cell Biol. Toxicol. 2001, 17, 301-317. [CrossRef]

14. Elsea, S.H.; Osheroff, N.; Nitiss, J.L. Cytotoxicity of quinolones toward eukaryotic cells. Identification of topoisomerase-II as the primary cellular target for the quinolone CP-115,953 in yeast. J. Biol. Chem. 1992, 267, 13150-13153. [PubMed]

15. Oliphant, C.M.; Green, G.M. Quinolones: A comprehensive review. Am. Fam. Physician 2002, 65, 455-464. [PubMed]

16. Bourikas, L.A.; Kolios, G.; Valatas, V.; Notas, G.; Drygiannakis, I.; Pelagiadis, I.; Manousou, P.; Klironomos, S.; A Mouzas, I.; Kouroumalis, E. Ciprofloxacin decreases survival in HT-29 cells via the induction of TGF- $\beta 1$ secretion and enhances the anti-proliferative effect of 5-fluorouracil. Br. J. Pharmacol. 2009, 157, 362-370. [CrossRef] [PubMed]

17. Zeng, X.H.; Li, Y.H.; Wu, S.S.; Hao, R.L.; Li, H.; Ni, H.; Han, H.B.; Lin, L. New and Highly Efficient Column Chromatographic Extraction and Simple Purification of Camptothecin from Camptotheca acuminata and Nothapodytes pittosporoides. Phytochem. Anal. 2013, 24, 623-630. [CrossRef] 
18. Arafa, R.K.; Hegazy, G.H.; Piazza, G.; Abadi, A.H. Synthesis and in vitro antiproliferative effect of novel quinoline-based potential anticancer agents. Eur. J. Med. Chem. 2013, 63, 826-832. [CrossRef]

19. Slaninova, I.; Pencikova, K.; Urbanova, J.; Slanina, J.; Taborska, E. Antitumour activities of sanguinarine and related alkaloids. Phytochem. Rev. 2013, 13, 51-68. [CrossRef]

20. Khalifa, N.; Eweas, A.; Al-Omar, M.A.; Hozzein, W.N. Synthesis and antimicrobial activity of some novel 8-hydroxy-7-iodoquinoline-5-sulfonamide derivatives. J. Pure Appl. Microbiol. 2014, 8, 629-637.

21. Kos, J.; Mitrovic, A. Nitroxoline: Repurposing its antimicrobial to antitumor application. Acta Biochim. Pol. 2019, 521-531. [CrossRef]

22. Zhao, C.; Chen, X.; Yang, C.; Zang, D.; Lan, X.; Liao, S.; Zhang, P.; Wu, J.; Li, X.; Liu, N.; et al. Repurposing an antidandruff agent to treating cancer: Zinc pyrithione inhibits tumor growth via targeting proteasome-associated deubiquitinases. Oncotarget 2017, 8, 13942-13956. [CrossRef]

23. Merriam, C.V.; Citron, D.M.; Tyrrell, K.L.; Warren, Y.A.; Goldstein, E.J. In vitro activity of azithromycin and nine comparator agents against 296 strains of oral anaerobes and 31 strains of Eikenella corrodens. Int. J. Antimicrob. Agents 2006, 28, 244-248. [CrossRef] [PubMed]

24. Kheadr, E.; Bernoussi, N.; Lacroix, C.; Fliss, I. Comparison of the sensitivity of commercial strains and infant isolates of bifidobacteria to antibiotics and bacteriocins. Int. Dairy J. 2004, 14, 1041-1053. [CrossRef]

25. Moubareck, C.A.; Gavini, F.; Vaugien, L.; Butel, M.J.; Doucet-Populaire, F. Antimicrobial susceptibility of bifidobacteria. J. Antimicrob. Chemother. 2005, 55, 38-44. [CrossRef] [PubMed]

26. Sharma, R.; Park, T.E.; Moy, S. Ceftazidime-Avibactam: A Novel Cephalosporin/ $\beta$-Lactamase Inhibitor Combination for the Treatment of Resistant Gram-negative Organisms. Clin. Ther. 2016, 38, 431-444. [CrossRef]

27. Novakova, J.; Dzunkova, M.; Musilova, S.; Vlkova, E.; Kokoska, L.; Moya, A.; D’Auria, G.; Unkova, M.D. Selective growth-inhibitory effect of 8-hydroxyquinoline towards Clostridium difficile and Bifidobacterium longum subsp. longum in co-culture analysed by flow cytometry. J. Med. Microbiol. 2014, 63, 1663-1669. [CrossRef]

28. Novakova, J.; Vlkova, E.; Salmonova, H.; Pechar, R.; Rada, V.; Kokoska, L. Anticlostridial agent 8-hydroxyquinoline improves the isolation of faecal bifidobacteria on modified Wilkins-Chalgren agar with mupirocin. Lett. Appl. Microbiol. 2016, 62, 330-335. [CrossRef]

29. Skrivanova, E.; Van Immerseel, F.; Hovorkova, P.; Kokoska, L. In Vitro Selective Growth-Inhibitory Effect of 8-Hydroxyquinoline on Clostridium perfringens versus Bifidobacteria in a Medium Containing Chicken Ileal Digesta. PLOS ONE 2016, 11, e0167638. [CrossRef]

30. Kim, Y.M.; Jeong, E.Y.; Lim, J.H.; Lee, H.S. Antimicrobial effects of 8-quinolinol. J. Food Sci. Biotechnol. 2006, 15, 817-819.

31. Prapasarakul, N.; Tummaruk, P.; Niyomtum, W.; Tripipat, T.; Serichantalergs, O. Virulence Genes and Antimicrobial Susceptibilities of Hemolytic and Nonhemolytic Escherichia coli Isolated from Post-Weaning Piglets in Central Thailand. J. Vet. Med. Sci. 2010, 72, 1603-1608. [CrossRef]

32. Tranter, R.W. The in vitro activity of halquinol against Vibrio cholerae. J. Trop. Med. Hyg. 1968, 71, 146-149.

33. Sobke, A.; Makarewicz, O.; Baier, M.; Bar, C.; Pfister, W.; Gatermann, S.; Pletz, M.; Forstner, C. Empirical treatment of lower urinary tract infections in the face of spreading multidrug resistance: In vitro study on the effectiveness of nitroxoline. Int. J. Antimicrob. Agents 2018, 51, 213-220. [CrossRef] [PubMed]

34. Chandler, C.J.; Segel, I.H. Mechanism of the Antimicrobial Action of Pyrithione: Effects on Membrane Transport, ATP Levels, and Protein Synthesis. Antimicrob. Agents Chemother. 1978, 14, 60-68. [CrossRef] [PubMed]

35. Lahiri, S.C.; Dutta, N.K. Berberine and chloramphenicol in the treatment of cholera and severe diarrhoea. J. Indian Med. Assoc. 1967, 48, 1-11.

36. Sack, R.B.; Froehlich, J.L. Berberine inhibits intestinal secretory response of Vibrio cholerae and Escherichia coli enterotoxins. Infect. Immun. 1982, 35, 471-475. [CrossRef]

37. Hamoud, R.; Reichling, J.; Wink, M.R. Synergistic antibacterial activity of the combination of the alkaloid sanguinarine with EDTA and the antibiotic streptomycin against multidrug resistant bacteria. J. Pharm. Pharmacol. 2014, 67, 264-273. [CrossRef]

38. Chukwudi, C.U.rRNA Binding Sites and the Molecular Mechanism of Action of the Tetracyclines. Antimicrob. Agents Chemother. 2016, 60, 4433-4441. [CrossRef] [PubMed] 
39. Hu, D.; Han, Z.; Li, C.; Lv, L.; Cheng, Z.; Liu, S. Florfenicol induces more severe hemotoxicity and immunotoxicity than equal doses of chloramphenicol and thiamphenicol in Kunming mice. Immunopharmacol. Immunotoxicol. 2016, 38, 472-485. [CrossRef] [PubMed]

40. Onoda, T.; Ono, T.; Dhar, D.K.; Yamanoi, A.; Nagasue, N. Tetracycline analogues (doxycycline and COL-3) induce caspase-dependent and -independent apoptosis in human colon cancer cells. Int. J. Cancer 2005, 118, 1309-1315. [CrossRef]

41. Dewdney, J.M. Effects of beta-lactam antibiotics on eukaryotic cells. Cell Boil. Toxicol. 1986, 2, 509-511. [CrossRef]

42. Eisenstein, B.I.; Schaechter, M. DNA and Chromosome Mechanics. In Schaechter's Mechanisms of Microbial Disease; Schaechter, M., Engleberg, N.C., DiRita, V.J., Eds.; Lippincott Williams \& Wilkins: Philadelphia, PA, USA, 2007; p. 28.

43. Hanaki, H.; Kuwahara-Arai, K.; Boyle-Vavra, S.; Daum, R.S.; Labischinski, H.; Hiramatsu, K. Activated cell-wall synthesis is associated with vancomycin resistance in methicillin-resistant Staphylococcus aureus clinical strains Mu3 and Mu50. J. Antimicrob. Chemother. 1998, 42, 199-209. [CrossRef]

44. Oliveri, V.; Vecchio, G. 8-Hydroxyquinolines in medicinal chemistry: A structural perspective. Eur. J. Med. Chem. 2016, 120, 252-274. [CrossRef] [PubMed]

45. Freitas, L.B.D.O.; Borgati, T.F.; Gil, R.F.; Ruiz, A.; Marchetti, G.M.; De Carvalho, J.E.; Da Cunha, E.F.; Ramalho, T.D.C.; Alves, R.B. Synthesis and antiproliferative activity of 8-hydroxyquinoline derivatives containing a 1,2,3-triazole moiety. Eur. J. Med. Chem. 2014, 84, 595-604. [CrossRef] [PubMed]

46. Lazar, V.; Ditu, L.-M.; Pircalabioru, G.G.; Gheorghe, I.; Curutiu, C.; Holban, A.M.; Picu, A.; Petcu, L.; Chifiriuc, M.C. Aspects of Gut Microbiota and Immune System Interactions in Infectious Diseases, Immunopathology, and Cancer. Front. Immunol. 2018, 9, 1830. [CrossRef] [PubMed]

47. Rajkovic, A.; Jovanovic, J.; Monteiro, S.; Decleer, M.; Andjelkovic, M.; Foubert, A.; Beloglazova, N.; Tsilla, V.; Sas, B.; Madder, A.; et al. Detection of toxins involved in foodborne diseases caused by Gram-positive bacteria. Compr. Rev. Food Sci. Food Saf. 2020, 19, 1605-1657. [CrossRef]

48. Clinical and Laboratory Standards Institute. Methods for Dilution Antimicrobial Susceptibility Tests for Bacteria that Grow Aerobically, 3rd ed.; Approved Standard; Clinical and Laboratory Standards Institute: Wayne, PA, USA, 2015.

49. Hecht, D.W. Antimicrobial agents and susceptibility testing: Susceptibility testing of anaerobic bacteria. In Manual of Clinical Microbiology, 7th ed.; Murray, P.R., Baron, E.J., Pfaller, M.A., Tenover, F.C., Yolken, R.H., Eds.; ASM Press: Washington, DC, USA, 1999; pp. 1555-1562.

50. Houdkova, M.; Rondevaldova, J.; Doskocil, I.; Kokoska, L. Evaluation of antibacterial potential and toxicity of plant volatile compounds using new broth microdilution volatilization method and modified MTT assay. Fitoterapia 2017, 118, 56-62. [CrossRef]

51. Mosmann, T. Rapid colorimetric assay for cellular growth and survival: Application to proliferation and cytotoxicity assays. J. Immunol. Methods 1983, 65, 55-63. [CrossRef]

52. Houdkova, M.; Urbanova, K.; Doskocil, I.; Rondevaldova, J.; Novy, P.; Nguon, S.; Chrun, R.; Kokoska, L. In vitro growth-inhibitory effect of Cambodian essential oils against pneumonia causing bacteria in liquid and vapour phase and their toxicity to lung fibroblasts. S. Afr. J. Bot. 2018, 118, 85-97. [CrossRef]

53. TIBCO Software Inc. Statistica, Data Analysis Software System, Version 13.0. Available online: http: //statistica.io (accessed on 30 September 2017).

(C) 2020 by the authors. Licensee MDPI, Basel, Switzerland. This article is an open access article distributed under the terms and conditions of the Creative Commons Attribution (CC BY) license (http://creativecommons.org/licenses/by/4.0/). 